\title{
Spontaneous emission control of silicon nanocrystals by silicon three-dimensional photonic crystal structure fabricated by self-aligned two-directional electrochemical etching method
}

\author{
Daihei Hippo $^{\text {a,e,*, }}$, Kei Urakawab ${ }^{\text {, Yoshishige Tsuchiya }}{ }^{\text {a,c,e }}$, Hiroshi Mizuta ${ }^{\text {c,d,e }}$, Nobuyoshi Koshida ${ }^{\text {b,e }}$, \\ Shunri Oda ${ }^{\mathrm{a}, \mathrm{e}}$ \\ a Quantum Nanoelectronics Research Center, Tokyo Institute of Technology, 2-12-1, O-okayama, Meguro-ku, Tokyo 152-8552, Japan \\ b Graduate School of Engineering, Tokyo University of Agriculture and Technology, Koganei, Tokyo 184-8588, Japan \\ ' School of Electronics and Computer Science, University of Southampton, Highfield, Southampton SO17 1BJ, UK \\ d Department of Physical Electronics, Tokyo Institute of Technology, 2-12-1, O-okayama, Meguro-ku, Tokyo 152-8552, Japan \\ e SORST-JST (Japan Science and Technology Agency), Kawaguchi, Saitama 332-0012, Japan
}

\section{A R T I C L E I N F O}

\section{Article history:}

Received 5 January 2009

Received in revised form 12 February 2009

Accepted 22 February 2009

\section{Keywords:}

3D photonic crystal

Silicon nanocrystals

Time-resolved photoluminescence

\begin{abstract}
A B S T R A C T
A silicon three-dimensional photonic crystal (3DPC) structure has been fabricated using a self-aligned, two-directional electrochemical etching method. The spectral component of the photoluminescence (PL) for silicon nanocrystals deposited on the 3DPC structures increase at $750 \mathrm{~nm}$ and slightly decrease at $800 \mathrm{~nm}$. Time-resolved PL measurements reveal that the radiative recombination lifetime of the silicon nanocrystals on 3DPC structures decreases at $750 \mathrm{~nm}$ and increases at $800 \mathrm{~nm}$ compared to those on a silicon substrate without 3DPC structures. We conclude that the spontaneous emission control of silicon nanocrystals has been observed using the 3DPC structures.
\end{abstract}

(C) 2009 Elsevier B.V. All rights reserved.

\section{Introduction}

Recently, fabrication of three-dimensional photonic crystals (3DPCs) has been reported [1-5]. In particular, silicon-based 3DPCs show great potential for hybrid opto-electronic circuits integrated with highly developed silicon microelectronics technologies at low cost. We propose a two-directional etching process for fabrication of silicon 3DPC. This process involves etching perpendicular to silicon substrate followed by etching parallel to silicon substrate [6]. For the practical opto-electronic integration, interactions between the 3DPC and active materials are important. However, there are only a few reports $[7,8]$ on experimental observation of such interactions. This is due to the fact that 3DPCs with a large photonic band gap (PBG) require a highly porous structure, making it difficult to place light emitters at defects in such structures. In this paper, we describe the inclusion of silicon nanocrystals as light emitters in the silicon 3DPC structure. Further, the interaction between the light emitted from the silicon nanocrystals and the silicon 3DPC structures has been investigated in detail.

\footnotetext{
* Corresponding author at: Quantum Nanoelectronics Research Center, Tokyo Institute of Technology, 2-12-1, O-okayama, Meguro-ku, Tokyo 152-8552, Japan.

E-mail address: d-hippo@neo.pe.titech.ac.jp (D. Hippo).
}

\section{Sample fabrication}

Previously we fabricated 3DPC by two-directional electrochemical etching method using rectangular parallel-piped etchpits formed by electron cyclotron resonance reactive ion etching (ECRRIE) prior to the anodizaton process [6]. The width of the space charge region was decreased and the field strength was increased around the etchpits in the silicon electrode. This resulted in higher tunneling probability of charge carriers and therefore higher local current density [9]. This effect becomes significant if the radius of curvature of the etchpit is smaller than the width of the space charge region.

In this work, we introduce inverse-pyramidal etchpits [10], in which the electric field is concentrated at the center of the etchpits. The radius of curvature of etchpits is much smaller than the surrounding regions. At first, an $n^{+}$Si wafer $(0.04-0.06 \Omega \mathrm{cm})$ was oxidized for $1 \mathrm{~h}$ at $1000^{\circ} \mathrm{C}$ to form a $\mathrm{SiO}_{2}$ layer on top of silicon substrate. The sample surface was then patterned by electron beam (EB) lithography, followed by ECR-RIE for $\sim 7 \mathrm{~min}$ to form shallow etchpits on the silicon substrate. Then the sample was immersed in $\mathrm{KOH}$ solution (20\%) for $1 \mathrm{~min}$ at $80^{\circ} \mathrm{C}$ to form inverse-pyramidal etchpits. The anodization process details can be found elsewhere [6].

Step 1. Fig. 1 shows the cross-sectional scanning electron microscope (SEM) images of (a) the whole sample after the first 


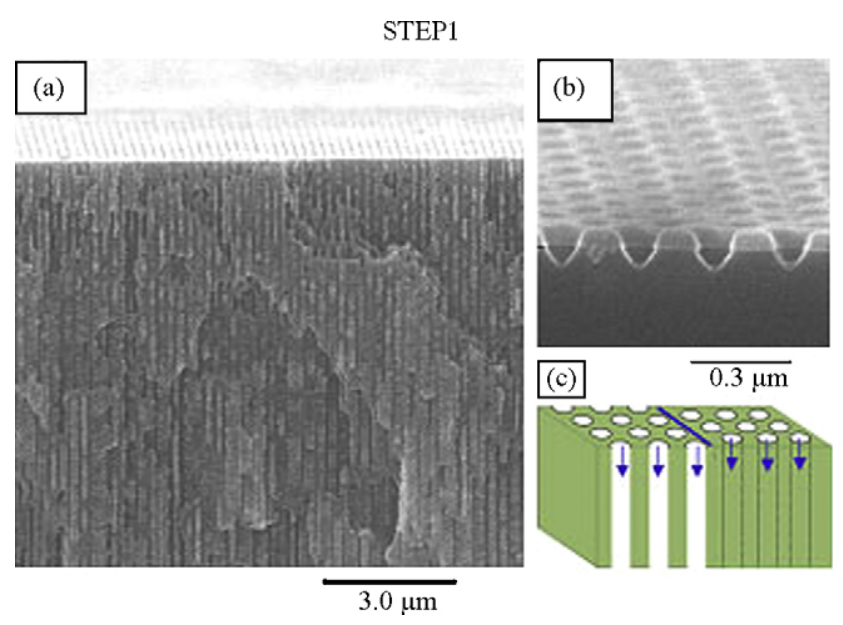

Fig. 1. Cross-sectional SEM images of (a) the whole sample after the first directional etching and (b) etchpits before the anodization process. (c) A schematic of the fabricated structure.

directional etching was performed, and (b) the inverse-pyramidal etchpits before the anodization. Fig. 1(c) shows a schematic of the fabricated sample structure. It should be noted that the phase of the EB patterns are shifted by half pitch at the straight line in the figure, and that after the formation of the inverse-pyramidal etchpits, no subsequent mask is required for pore growth [10].

Step 2 . In the second process, the structure is oxidized to fill the pores with $\mathrm{SiO}_{2}$, and a slope is formed along the straight boundary by mechanical polishing. At this stage, the patterning of pores on the slope is realized, as shown in Fig. 2. We can clearly observe a single defect on the upper part of the slope pattern, indicated by the circle in Fig. 2(a). Although the sample surfaces were not masked, the silicon surfaces at the defect sites were not etched at all during the electrochemical anodization processes. This is due to a structurally induced etchstop mechanism. During the anodization processes, the electric field is concentrated on sharp etchpits. Holes, which are responsible for initiating the electrochemical dissolution of silicon, are effectively collected at these sites. As a result, the defect sites become depleted of holes, and no dissolution occurs. It should be noted that the controlled etching is realized up to around $5 \mu \mathrm{m}$ in depth in the present state, since the structural fluctuation increases with the increase in etching time owing to the change in the ion concentration of the electrolyte along the pores, as shown in Fig. 2(b). Fig. 2(c) shows an SEM image of the whole sample. Fig. 2(d) shows a schematic of the fabricated sample structure.

Step 3. At the final stage, the second directional etching is performed in the direction perpendicular to the first directional etching using the pattern formed on the slope as a guide. A cleaved facet of the fabricated sample structure is shown in Fig. 3(a). The higher magnification image in Fig. 3(b) shows the layered structure of the slope pattern, with a period of approximately $200 \mathrm{~nm}$. This structure is designed for the central wavelength of the photonic band gap at around $800 \mathrm{~nm}$ [6], which is the typical luminescence peak of the nanocrystalline silicon [11]. The black circle in Figs. 2(a) and 3(b) indicate the defect introduced into the 3DPC.

\section{Optical measurements}

\subsection{Photoluminescence (PL)}

We deposited silicon nanocrystals on the 3DPC structures for PL measurements. Spherical-shaped silicon nanocrystals were deposited using a pulsed gas remote-plasma-enhanced chemical vapor deposition method [12]. The silicon nanocrystals have a mean diameter of $\sim 10 \mathrm{~nm}$, and a natural surface oxide. After deposition of the silicon nanocrystals, the sample was thermally oxidized for $8 \mathrm{~h}$ at $800^{\circ} \mathrm{C}$ to reduce the silicon core size. Visible photoluminescence centered at around $730 \mathrm{~nm}$ was observed owing to the quantum

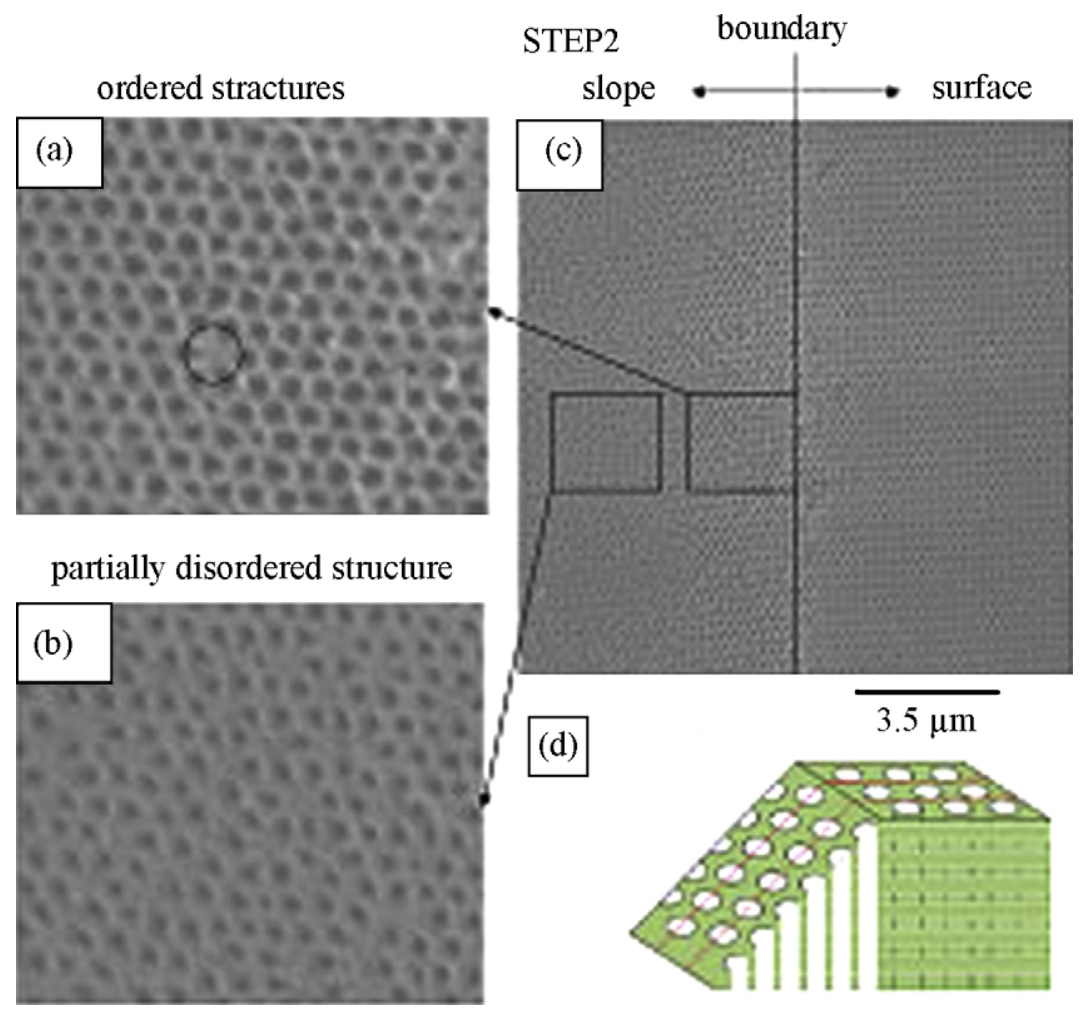

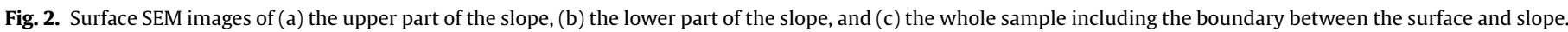
(d) A schematic of the fabricated structure. 


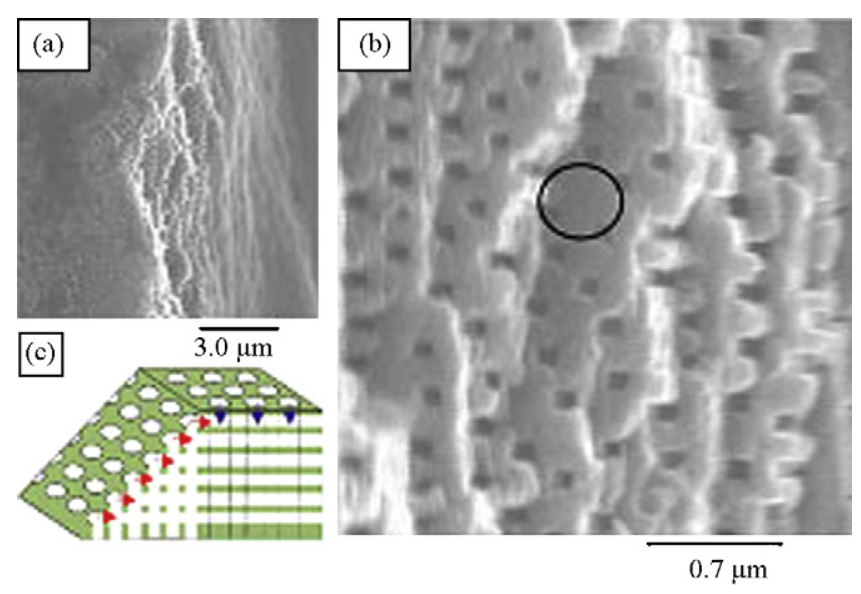

Fig. 3. Surface SEM images of (a) the cleaved facet of the fabricated $3 D P C$ and (b) its magnified view. A black circle indicates a defect introduced into the 3DPC. (c) A schematic of the fabricated 3DPC structure.

confinement effect [11]. It should be noted that the oxidation process also affects the photonic crystal structures because the large surface areas are exposed to the oxygen atmosphere. However, the initial diameter of the pores is designed for $\sim 50 \%$ of the optimized parameter to take this effect into account. The oxidation rate is approximately $3 \mathrm{~nm} \mathrm{~h}^{-1}$ at $800^{\circ} \mathrm{C}$ for a $\left(\begin{array}{lll}1 & 0 & 0\end{array}\right)$ silicon substrate.

PL measurements were performed using a He-Cd laser (442 nm, $5 \mathrm{~mW}$ ) at room temperature. The incident angle was $45^{\circ}$ and $\mathrm{PL}$ was detected in the direction perpendicular to the sample surface. The observed PL spectra are shown in Fig. 4. The central wavelength of the PL without 3DPC structure is $\sim 730 \mathrm{~nm}$ which corresponds to the silicon core diameter of about $4.5 \mathrm{~nm}$ [11]. To analyze the change in the spectral component, we derived the relative PL intensity of these two spectra, as shown in the inset in Fig. 4. Here spectral component centered at around $700 \mathrm{~nm}$ is increased on the 3DPC compared to that on the silicon substrate without 3DPC structures, while the component at around $800 \mathrm{~nm}$ is slightly suppressed. Specifically, the latter fact indicates the existence of the small photonic band gap in the 3DPC structures, while the former can be explained either by the enhancement of the light extraction efficiency caused by the surface roughness or by the increase in the coupling efficiency between the excited carriers in the silicon nanocrystals and the defect modes in the 3DPC structures. In these small area measurements in the order of $\mu \mathrm{m}^{2}$, it is critical to distinguish the surface effects from those induced by the 3DPC structures to identify the origin of the changes in the reflectance and the PL.

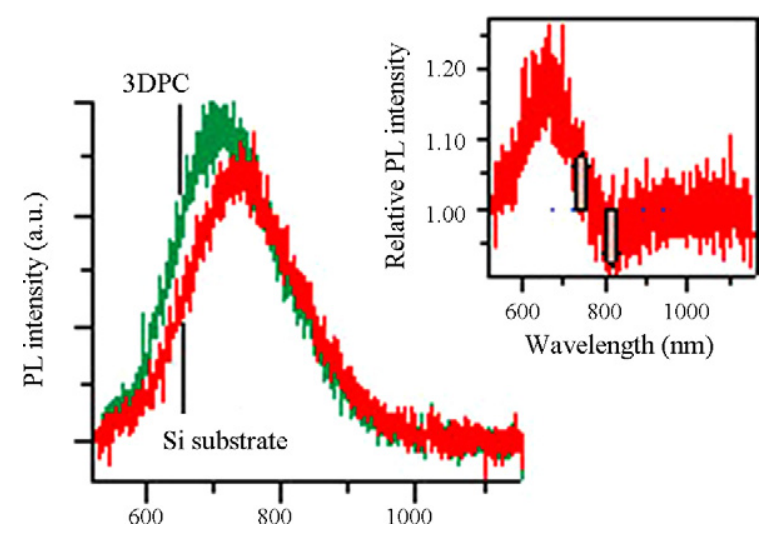

Fig. 4. The PL spectra of silicon nanocrystals deposited onto the 3DPC and silicon substrate without 3DPC structures. The inset shows the relative PL intensity of silicon nanocrystals deposited onto the 3DPC normalized by that of silicon substrate without 3DPC structures.

(a) $3 \mathrm{DPC}$

(b) Si substrate

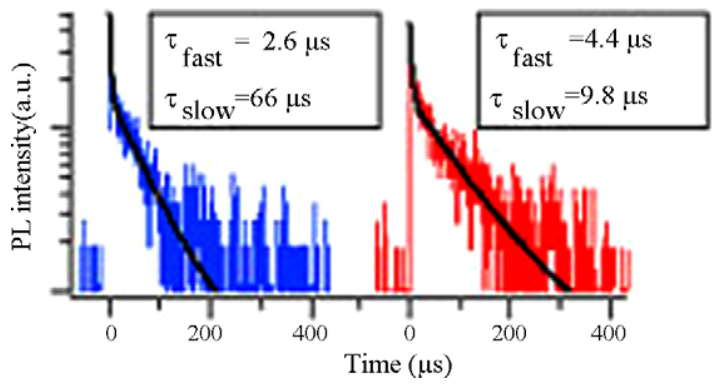

Fig. 5. The PL decay curves of silicon nanocrystals (a) on top of 3DPC and (b) on a silicon substrate measured at $750 \mathrm{~nm}$. The fitted curves are indicated as solid lines in the figures. The vertical axis is plotted in log scale.

\subsection{Time-resolved PL}

Time-resolved PL is independent of surface effects and so considered to be the most suitable way to understand the underlying mechanism for the above-mentioned experimental results. We performed the time-resolved PL measurements using the same sample to identify the origin of the changes in the spectral component in the PL in Fig. 4. The experiments were performed at room temperature, using yttrium aluminum garnet (YAG) laser $(532 \mathrm{~nm}$, $0.75 \mathrm{~mW}, 2 \mathrm{kHz}$ ), with a $\sim 30 \mu \mathrm{m}^{2}$ spot size. The measurements were performed at 750 and $800 \mathrm{~nm}$, corresponding to the wavelengths where the PL is enhanced and suppressed, respectively. The following double exponential function (Eq. (1)) is fitted to each of the experimental decay curve:

$I=I_{0}+A \exp \left(-\frac{t}{\tau_{\text {fast }}}\right)+B \exp \left(-\frac{t}{\tau_{\text {slow }}}\right)$

where $I$ and $I_{0}$ represent PL intensity, $A$ and $B$ are constants, the variable $t$ represents time, $\tau_{\text {fast }}$ and $\tau_{\text {slow }}$ are the lifetime of the fast and slow decay processes, respectively. Fig. 5 shows the experimental results of the decay curves of silicon nanocrystals measured at a fixed wavelength of $750 \mathrm{~nm}$ : (a) on top of 3DPC and (b) on a silicon substrate without 3DPC structures.

In the case of 3DPC, $\tau_{\text {fast }}=2.6 \mu \mathrm{s}$ and $\tau_{\text {slow }}=66 \mu \mathrm{s}$, while for the silicon substrate, $\tau_{\text {fast }}=4.4 \mu \mathrm{s}$ and $\tau_{\text {slow }}=98 \mu \mathrm{s}$. These values indicate that the decay times of both the fast and the slow components decrease by a factor of 1.5 on 3DPC compared to those on the silicon substrate. Fig. 6 shows the decay curves of the same sample measured at a fixed wavelength of $800 \mathrm{~nm}$. For the 3DPC, $\tau_{\text {fast }}=3.5 \mu \mathrm{s}$ and $\tau_{\text {slow }}=78 \mu \mathrm{s}$, while for the silicon substrate, $\tau_{\text {fast }}=2.4 \mu \mathrm{s}$ and $\tau_{\text {slow }}=87 \mu \mathrm{s}$. In this case, the decay time of the fast component increases by a factor of 1.5 and that of the slow component decreases

(a) $3 \mathrm{DPC}$

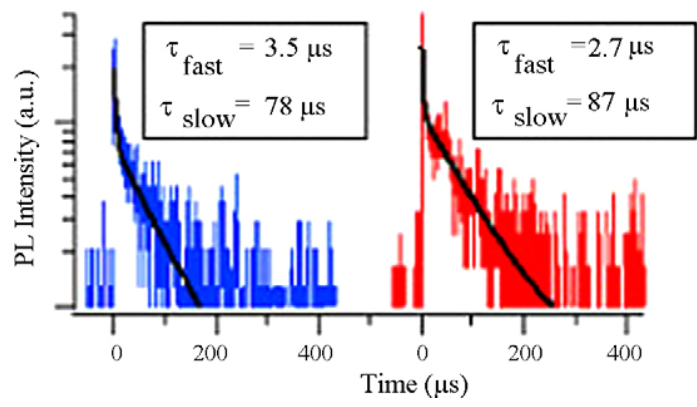

Fig. 6. The PL decay curves of silicon nanocrystals (a) on top of 3DPC and (b) on a silicon substrate measured at $800 \mathrm{~nm}$. The fitted curves are indicated as solid lines in the figures. The vertical axis is plotted in log scale. 
(a)
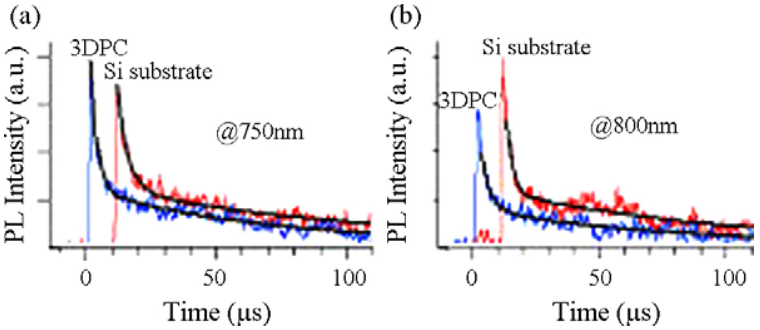

Fig. 7. Magnifications of the decay curves that mainly include fast components (a) in Fig. 5 and (b) in Fig. 6. The vertical axis is plotted in linear scale.

by a factor of 1.1 on 3DPC as compared to that on the silicon substrate.

\section{Discussion}

Strictly speaking, the observed lifetimes are not identical to the intrinsic radiative recombination lifetimes because they are determined by the both radiative and nonradiative recombination processes. The intrinsic radiative lifetime $\tau_{R}$, can be obtained from the observed lifetime $\tau_{\mathrm{obs}}$ and PL intensity $I_{\mathrm{PL}}$, as indicated in Eq. (2) [13].

$\tau_{\mathrm{R}} \propto \frac{\tau_{\mathrm{obs}}}{I_{\mathrm{PL}}}$

Based on these relationships, we analyzed both PL intensity and the observed lifetimes. Fig. 7(a) shows the magnification of the fast components of the PL decay curves from Fig. 5, while Fig. 7(b) shows those from Fig. 6 . At $750 \mathrm{~nm}$, PL intensity increases by a factor of 1.2 at $t=0$. It should be noted that the integrated PL intensity, which mainly include fast components, is approximately proportional to the PL intensity at $t=0$. The observed lifetime decreases by a factor of 1.5 on 3DPC so the intrinsic radiative recombination lifetime also decreases according to Eq. (2). Similarly, at $800 \mathrm{~nm}$, PL intensity decreases by a factor of 1.4 , and the observed lifetime increases by a factor of 1.5 on 3DPC. Hence, the intrinsic radiative recombination lifetime also increases. These results are consistent with the changes in PL spectra in Fig. 4. Thus, we can conclude that spontaneous emission control of Si nanocrystals has been realized using the 3DPC structures for the fast components. On the other hand, the lifetimes of the slow components decrease on 3DPC both at 750 and $800 \mathrm{~nm}$ by factors of 1.5 and 1.1, respectively. Meanwhile, the PL intensity of these components decrease significantly, as shown in Figs. 5 and 6. Thus, the behavior of the slow components is considered to be governed by nonradiative processes. The mechanism for the nonradiative decay comes from an escape of the carriers from the confined zone to more extended or disordered regions where nonradiative recombination can occur [14]. It should be noted that about $80 \%$ of the PL consists of fast components for both decay curves. The fast components, which are of the order of $\mu \mathrm{s}$, are considered to originate from inside the silicon nanocrystals, where the oscillator strength for the transition is increased compared to that of bulk silicon. In comparison, the slow components, which occur over several tens of $\mu$, are considered to originate from the interfacial layers between silicon nanocrystals and their surrounding oxide $[14,15]$, where carrier density tends to increase and the effects of nonradiative processes should be dominant. Silicon nanocrystals are deposited both on the surface of 3DPC and inside the pores. The changes in PL decay times should only occur inside the 3DPC pores, and hence the 3DPC structures affect only small parts of the deposited silicon nanocrystals, resulting in the small observed changes in PL characteristics.

There are two remaining issues to be discussed about the changes in PL decay times. The integrity of the fabricated structure may not be sufficient to realize a significant Purcell effect in the present state, and also, the absorption effect cannot be neglected in the wavelength range of interest. Therefore, the observed reduction in PL decay time in Fig. 5 could be interpreted not only as the Purcell effect induced by a photonic band gap effect but also as structurally induced changes in the spatial overlap of carriers and cavity modes. However, the fact that we have successfully demonstrated spontaneous emission control of Si nanocrystals by the 3DPC structures suggests that it is possible to realize the Purcell effect by improving the fabrication processes. Concerning the absorption effect, the effective absorption coefficient of the mesoporous silicon is reduced because of the high porosity, and the absorption edges are blue shifted compared with that of bulk silicon [16]. Although the free carriers in heavily doped silicon absorb photons below the fundamental band gap [17], if such substrates are made mesoporous by anodization and subsequently dried, the absorption coefficient decreases by orders of magnitude with increasing porosity [18]. The decreased absorption coefficient for the infrared regime in mesoporous silicon has been interpreted to be caused by the trapping of free charge carriers at deep levels related to the surface states [19]. These two effects are enhanced by increasing the porosity, resulting in a very low absorption for the highly porous 3DPC structures.

\section{Conclusions}

We fabricated a silicon three-dimensional photonic crystal structure using the self-aligned two-directional electrochemical etching method. Optical measurements were performed to determine the properties of the fabricated 3DPC structures. For silicon nanocrystals deposited on the 3DPC structures, we observed that the spectral component of the PL was increased at $750 \mathrm{~nm}$ and slightly decreased at $800 \mathrm{~nm}$. Time-resolved PL measurements reveal that each PL decay curve consists of a fast and a slow component. The decay time of the fast component decreases at $750 \mathrm{~nm}$ and increases at $800 \mathrm{~nm}$ on 3DPC structures compared to those on the silicon substrate without 3DPC structures. This is attributed to radiative recombination processes, while the behavior of the slow components is attributed to nonradiative processes. Thus, we can conclude that spontaneous emission control of silicon nanocrystals has been realized using 3DPC structures, for the fast components of the decay. These results reveal the advantage of silicon nanocrystals fabricated by a pulsed gas remote-plasma-enhanced chemical vapor deposition method compared to other silicon nanostructures such as porous silicon and ion-implanted silicon nanocrystals in which nonradiative contributions are dominant. Silicon nanocrystals, combined with highly controlled photonic crystal structures, are promising candidates as light emitters for future opto-electronic integration.

\section{Acknowledgments}

The authors would like to thank Dr. Y. Ogawa for the technical assistance in the time-resolved PL measurements and Dr. K. Usami for the deposition of silicon nanocrystals.

\section{References}

[1] J. Schilling, J. White, A. Scherer, G. Stupian, R. Hillebrand, U. Gosele, Appl. Phys. Lett. 86 (2005) 011101.

[2] S. Noda, K. Tomoda, N. Yamamoto, A. Chutinan, Science 289 (2000) 604

[3] M. Qi, E. Lidorikis, P.T. Rakish, S.G. Johnson, J.D. Joannopoulos, E.P. Ippen, H.I Smith, Nature 429 (2004) 538.

[4] S. Matthias, F. Muller, C. Jamois, R.B. Wehrspohn, U. Gosele, Adv. Mater. 16(2004) 2166.

[5] N. Tetreault, G.V. Freymann, M. Deubel, M. Hermatschweiler, F. Perez-Willard, S. John, M. Wegener, G.A. Ozin, Adv. Mater. 18 (2006) 457.

[6] D. Hippo, K. Urakawa, Y. Kawata, Y. Tsuchiya, H. Mizuta, N. Koshida, S. Oda, Jpn. J. Appl. Phys. 46 (2007) 633. 
[7] M. Megens, J.E.G.J. Wijnhoven, A. Lagendijk, W.L. Vos, Phys. Rev. A 59 (1999) 4727.

[8] S. Noda, M. Imada, M. Okano, S. Ogawa, M. Mochizuki, A. Chutinan, IEEE J. Quantum Electron. 38 (2002) 726.

[9] X.G. Zhang, J. Electrochem. Soc. 138 (1991) 3750.

[10] V. Lehmann, H. Föll, J. Electrochem. Soc. 137 (1990) 653.

[11] K. Arai, J. Omachi, K. Nishiguchi, S. Oda, Mater. Res. Soc. Symp. Proc. 664 (2001) A20.6.1.

[12] T. Ifuku, M. Otobe, A. Itoh, S. Oda, Jpn. J. Appl. Phys. 36 (1997) 4031.
[13] S. Fukatsu, Y. Mera, M. Inoue, K. Maeda, Thin Solid Films 294 (1997) 33

[14] J.C. Vial, A. Bsiesy, F. Gaspard, R. Herino, M. Ligeon, F. Muller, R. Romestain, Phys. Rev. B 45 (1992) 14171.

[15] L. Pavesi, L. Dal Negro, C. Mazzoleni, G. Franzo, F. Priolo, Nature 408 (2000)440.

[16] D. Kovalev, G. Polisski, M. Ben-Chorin, J. Diener, F. Koch, J. Appl. Phys. 80 (1996) 5978.

[17] R.A. Soref, B.R. Bennett, IEEE J. Quantum Electron. 23 (1997) 123.

[18] M.H. Chan, S.K. So, K.W. Cheah, J. Appl. Phys. 79 (1996) 3273.

[19] V. Lehmann, F. Hofmann, F. Möller, U. Grüning, Thin Solid Films 255 (1995) 20. 